\title{
Kernos
}

Revue internationale et pluridisciplinaire de religion grecque antique

8 | 1995

Varia

\section{Influences orientales dans la littérature grecque : quelques réflexions de méthode}

\section{Alberto Bernabé}

\section{OpenEdition \\ Journals}

Édition électronique

URL : http://journals.openedition.org/kernos/581

DOI : 10.4000/kernos.581

ISSN : 2034-7871

Éditeur

Centre international d'étude de la religion grecque antique

Édition imprimée

Date de publication : 1 janvier 1995

Pagination : 9-22

ISSN : 0776-3824

Référence électronique

Alberto Bernabé, «Influences orientales dans la littérature grecque : quelques réflexions de méthode », Kernos [En ligne], 8 | 1995, mis en ligne le 11 avril 2011, consulté le 02 mai 2019. URL : http:// journals.openedition.org/kernos/581; DOI : 10.4000/kernos.581 
Kernos, 8 (1995), p. 9-22.

\section{Influences orientales dans la littérature grecque : quelques rëflexions de mëthode}

1. Les découvertes de plus en plus importantes de documents littéraires issus de diverses cultures du Proche-Orient ainsi que les progrès dans l'étude des langues dans lesquelles ceux-ci sont écrits ont eu pour résultat une connaissance plus approfondie d'une littérature extrêmement féconde dont l'accès nous était demeuré jusqu'à présent interdit. L'approche de textes littéraires du Proche-Orient antique a très tôt permis d'y déceler des ressemblances, souvent très évidentes, avec des œuvres grecques, notamment dans le domaine des mythes. De tels progrès ouvrent de nouvelles voies dans l'approche de la littérature grecque, dans la mesure où celle-ci va de ce fait s'insérer dans un autre contexte; elle nous oblige cependant à nous interroger sur la façon dont ces influences ont pu s'exercer ainsi que sur leur portée.

Les réponses qui ont pu être fournies à ces questions sont extrêmement variées et parfois même contradictoires : il y a ceux qui considèrent la littérature grecque comme une littérature orientale parmi d'autres (à ce sujet, il faut rappeler la phrase provocatrice de West ${ }^{1}$, « Greece is part of Asia : Greek literature is a Near Eastern literature ») et qui ont donc relevé d'innombrables parallélismes, il y a ceux qui croient que la littérature grecque n'est strictement qu'un produit hellénique, à l'abri de toute influence extérieure vraiment significative. Parmi les différentes raisons servant à justifier une telle disparité nous pouvons en signaler une : on n'est pas encore arrivé à mettre en place une méthodologie suffisamment élaborée qui permette d'aborder les emprunts littéraires et qui soit capable d'aller au-delà du simple constat des ressemblances. Dans ce sens, d'énormes progrès ont été accomplis, comme en témoignent les recherches de Burkert ${ }^{2}$, Mondi $^{3}$, Motte et Pirenne-Delforge ${ }^{4}$ qui ont par ailleurs beaucoup contribué à cette étude.

1 M. L. WEST, Hesiod Theogony, Oxford, 1966, p. 31.

2 W. BURKERT, Itinerant deviners and magicians: A neglected element in cultural contacts, in R. HÄGG (ed.), The Greek Renaissance of the Eightb Century B. C. Tradition and Innovation, Stockholm, 1983, p. 115-120; Die orlentalisierende Epocbe in der griecbischen Religion und 
L'objet de ce travail consistera à fournir quelques réflexions méthodologiques sur l'étude des influences orientales ${ }^{5}$ dans les textes grecs, notamment dans le domaine des textes mythologiques, forcément sommaires, étant donné qu'une analyse approfondie exigerait bien plus de temps. Je me limiterai donc à soulever quelques questions fondamentales, accompagnées de quelques exemples, tirés essentiellement des rapports entre des textes hittites et grecs, étant donné que c'est dans ce domaine que je me sens le plus à l'aise.

2. La question est hêrissée de difficultés. Celles qui méritent d'être signalées sont les suivantes :

2.1. Le premier problème se pose au moment de trancher la question de savoir s'il existe ou non des emprunts ${ }^{6}$ entre les deux littératures. Il est cependant relativement aisé de déceler des ressemblances entre différents textes, non seulement entre la littérature grecque et les littératures orientales, mais encore entre d'autres littératures du monde, quelle que soit leur nature. On pourrait même dire qu'il s'agit là de quelque chose de trop facile, ce qui explique qu'on ait souvent eu tendance à relever ces possibles emprunts avec une certaine légèreté. C'est ainsi que pendant assez longtemps on a considéré qu'un bref texte hittite, dans lequel apparaissait le héros Gourparanzahhu participant à une compétition de tir à l'arc, représentait de toute évidence un antécédent de la fameuse épreuve de l'arc que nous retrouvons dans L'Odyssée d'Homère. Une analyse plus poussée, réalisée par Güterbock ${ }^{7}$, a bien mis en évidence que les similitudes étaient assez lointaines. Et ne parlons pas des réactions soulevées par un prétendu poème louvite sur la guerre de Troie, une Wilousiade, qui aurait été un antécédent de L'Iliade homérique. L'existence de

Literatur, Heidelberg, 1984 (The Orientalizing revolution, Cambridge Mass., 1992); Ortental and Greek Mytbology: The meeting of parallels, in J. BREMMER (ed.), Interpretations of Greek Mythology, London and Sydney, 1987, p. 10-40.

3 R. MONDI, Greek mythic thought in the light of the Near East, in L. EDMUNDS (ed.), Approaches to Greek myth, Baltimore and London, 1990, p. 142-198.

4 A. MOTTE, V. PIRENNE-DELFORGE, Du "bon usage" de la notion de syncrétisme, in Kernos, 7 (1994), p. 11-27.

5 Cf. W. BURKERT, Oriental and Greek...art. cit. (n. 2), p. 12-3: "The term 'oriental' in itself... is a label that all too clearly echoes the ethnocentric perspective of 'Westerners' and tends to obscure the fact that quite different civilisations existed more or less to the east, or the southeast, of Europe".

6 Sur la notion d'emprunt, cf. MOTTE, PIRENNE-DELFORge, art. cit. (n. 4), p. 13 sq.

7 H.G. GÜTERBOCK, Die bistoriscbe Tradition und ibre Literarische Gestaltung bei Babylontern und Hetbitern bis 1200, II, Hetbiter; in ZAss, 44 (1938), p. 45-149, esp. p. 83 sq. 
cet hypothétique poème n'était fondée que sur un incipit mentionné entre les «mots sacrés» qui devaient être chantés dans un rituel istanouwien ${ }^{8}$.

2.2. La seconde difficulté à laquelle nous nous heurtons et que nous devons signaler réside dans le fait que le seul moyen dont nous disposons pour avoir accès à la littérature grecque et aux littératures orientales se trouve dans leurs manifestations écrites. Il est cependant presque certain que, dans la plupart des cas, le poète grec n'a pu avoir accès à la source écrite orientale que nous connaissons.

En effet, il existe un écart temporel évident entre l'apogée de la plupart des littératures du Proche-Orient, qui doit se situer dans le second millénaire av. J.-C., et les débuts de la littérature grecque connue qui ne remontent pas au-delà du VIIIe siècle. Il faut toutefois insister sur les différences concernant la transmission de la littérature grecque par rapport à la transmission des littératures orientales, telles que nous les connaissons. Les littératures procheorientales, comme chacun sait, sont exclusives des milieux de cour et se trouvent entre les mains d'un groupe de personnes qui connaissent l'écriture, les scribes. Ces vrais érudits, qui devaient connaître des langues étrangères afin de faciliter les travaux de la chancellerie et les relations internationales entre les rois au service desquels ils étaient, non seulement traduisaient des traités et des lettres officielles, mais aussi de la littérature; la preuve en est que nous retrouvons des textes littéraires sur Gilgamesh; dans des territoires situés ailleurs qu'en Mésopotamie (comme Hatti ou Ebla). Dans le monde grec, les seuls scribes connus dans le second millénaire av. J.-C. sont ceux des palais mycéniens, qui n'ont jamais écrit de textes littéraires, tandis que tout laisse supposer que la littérature, qui ne serait écrite que bien plus tard, était transmise à cette époque-là oralement. Il est donc pratiquement impossible que les mythes orientaux soient parvenus en Grèce par le biais de la traduction de textes écrits ${ }^{9}$. Je parle évidemment d'une façon générale, il s'agit d'une tout autre question quand on parle de Bérose, de l'hypothétique accès de Philon de

8 Voici l'inciplt: abhatata alatt awtenta wilusatt " quand ils vinrent de l'escarpée (?) Wilusa ", cf. C. WATKINS, The language of Trofans, in M. J. MELLINK (ed.), Troy and the Trojan Ward. A Sympostum beld at Bryn Mawr College, October 1984, Bryn Mawr, 1986, p. 45-62; Y.L. ARBEITMAN, Trofan, Luwian and the mass medla, 1985 (c.e.), in Diacbronica III 2 (1986), p. 283291; A. BERNABÉ, Mitología bitita, in G. DEL OLMO LETE (ed.), Mitología del próximo ortente, III, Sabadell, sous presse.

9 Cf. J. DE HOZ, Hesíodo en sociedad, in J.A. LÓPEZ FÉREZ (ed.), La épica grega y su influencia en la literatura española, Madrid, Ediciones Clásicas, 1994, p. 113-154, esp. p. 125-128. 
Byblos à des textes phéniciens écrits ${ }^{10}$ ou du possible contact de voyageurs grecs avec des textes égyptiens qui leur avaient été traduits.

Nous retrouvons cependant des empreintes évidentes d'œuvres orientales dans la littérature grecque à des époques très tardives. Pour citer un exemple bien connu, Oppien ${ }^{11}$ nous rapporte que Pan de Kôrykos attira trompeusement le monstre Typhon vers la terre avec la promesse d'un banquet de poisson, afin que Zeus pût le vaincre, et Nonnos ${ }^{12}$ nous raconte comment ce même personnage avait enlevé à Zeus les tendons, et comment Cadmos réussit à le séduire avec la musique et les lui demanda, sous prétexte de les utiliser comme cordes pour sa lyre. Ces deux récits présentent de grandes ressemblances par rapport aux textes écrits du vieux mythe hittite de la lutte contre le Dragon qui servaient de base au culte de Nerik ${ }^{13}$; mais quand Oppien ou Nonnos écrivirent leurs œuvres, les textes hittites auxquels elles ressemblent étaient ensevelis sous terre depuis plus de dix siècles et les derniers à parler la langue hittite avaient de même disparu depuis longtemps.

2.3. Ceci nous montre qu'il y a eu vraisemblablement d'autres voies de transmission que l'écriture. Au moment d'envisager l'hypothèse de ces voies alternatives, celles qui nous viennent le plus immédiatement à l'esprit sont la voie orale et celle des images, sans qu'il y ait entre elles aucune incompatibilité.

Or, le fait de parler d'une transmission orale ne résout toujours pas le problème, car il n'est pas non plus évident qu'un poète oriental puisse aller en Grèce chanter des poèmes dans sa propre langue, et encore moins, en grec, La transmission orale a dû certainement avoir lieu par l'intermédiaire de voyageurs ayant eu oralement accès à des récits orientaux - et non, répétons-le, à des versions écrites, monopole des scribes, - à la façon des contes populaires qui voyagent, parcourant l'Europe entière et franchissant les barrières linguistiques. 1981.

10 Cf. A.I. Baumgarten, The 'Phoenician bistory' of Philo of Byblos. A commentary, Leiden,

11 OPPIEN, Hal., III, 15-25.

12 NONNOS, Dion., I, 481 sq.

13 W. PORZIG, Illuyankas und Typbon, in Klelnasiatiscbe Forschungen, 1 (1930), p. 379-386; F. VIAN, Le mythe de Typhée et le problème de ses origines orientales, in Éléments ortentaux dans la religion grecque anclenne, Paris, 1960, p. 17-37; J. FONTENROSE, Typhon among the Arimol, in L. WALLACH (ed.), The classical tradition, literary and bistortcal studies in bonor of $H$. Caplan, Ithaca Nueva York, 1966, p. 64-82; ID., Pytbon, Berkeley, $1980^{2}$; W. BURKERT, Propp's beritage and Illuyankas, in Structure and bistory in Greek Mytbology and ritual, Berkeley, 1979; G. BECKMAN, The anatolian myth of Illuyanka, in JANES, 14 (1982), p. 11-25; A. BERNABÉ, La lucha contra el Dragón: el viaje de un mito, in J. MENDOZA (ed.), Mediterráneo y antiguo ortente próximo: contactos culturales, Curso de Verano de la Universidad Complutense en Almería, 22-26 julio 1991, sous presse. 
Une telle transmission est incompatible avec des versions longues et élaborées sur le plan littéraire, de même qu'elle peut être à l'origine de profondes altérations concernant les textes.

Quant aux images, elles sont d'une grande importance, car non seulement elles nous confirment qu'un thème mythique était connu en Grèce à une certaine date mais aussi elles nous fournissent un mode de transmission du mythe : l'explication de l'histoire qui était racontée par l'image. Une réponse aux questions « Qui est ce personnage et que fait-il ? ", à propos de l'image qui apparaît sur une pièce importée, peut constituer une voie importante de pénétration de thèmes mythiques dans une culture étrangère.

Il existerait aussi d'autres possibilités. Ainsi Burkert ${ }^{14}$ suggérait-il l'hypothèse des devins et des conjurateurs, notamment ceux qui réalisaient certains rites liés à des pratiques magiques.

Le problème est extrêmement complexe. Nous connaissons quelques textes écrits des littératures orientales auxquels les Grecs n'ont pas eu accès. Et les Grecs, à leur tour, ont certainement eu accès à des variantes de ces récits, transmises oralement, que nous ne connaissons pas. Uniquement dans le monde des images il nous est parfois possible de suivre les traces de certains thèmes, que nous trouvons sur des pièces orientales et sur des objets artistiques grecs et qui rendent incontestable l'existence d'emprunts. Burkert nous a apporté quelques exemples extrêmement intéressants de ce genre d'influences ${ }^{15}$.

2.4. Si nous admettons que les contacts entre les littératures orientales et la littérature grecque ont dû être foncièrement oraux, nous devons au moins établir comme hypothèse le lieu et l'époque où ceux-ci se sont vraisemblablement produits. Les lieux sont parfois évidents. Dans l'exemple cité auparavant, Oppien est de Cilicie; or le mythe du Dragon est depuis toujours lié à la zone. Pour citer un autre exemple de l'évidence du lieu, des vers de Nonnos $^{16}$, qui expliquent la raison du nom de la cordillère du Taurus, peuvent être mis en rapport avec un mythe hittite nous rapportant comment un dieu transformé en taureau ouvrit le passage à ses troupes dans cette

14 W. BURKER'T, Itinerant deviners... art. cit. (n. 2); Die orientalisierende Epocbe... op. cit. (n. 2), p. 43-84,

15 W. BURKERT, Oriental and Greek... art. cit. (n. 2), p. 25-30.

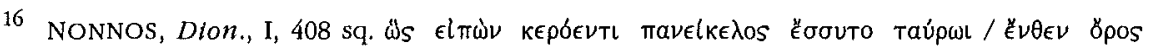

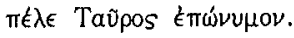


cordillère $^{17}$. Il doit s'agir bien évidemment d'un mythe associé à un lieu précis, le Taurus, visant à justifier son nom. Dans des cas comme ceux-ci, il est permis de supposer qu'il est question de mythes locaux recueillis sur place et qui dépassent la superposition des différentes cultures ${ }^{18}$. Il est toutefois évident que l'identification du lieu de l'emprunt n'est possible que dans des cas particulièrement favorables; dans la plupart des cas on relève des ressemblances, mais on ne cesse de rencontrer des obstacles qui rendent difficile ou impossible le repérage du lieu où a pu se produire le contact.

2.5. Quant à l'époque, on a signalé deux moments spécialement favorables pour les contacts de la littérature grecque avec les littératures orientales : l'âge du bronze tardif (XIVe-XIIIe siècle av. J.-C., époque que Gordon a désignée sous le nom de Aegean Koine ${ }^{19}$ et l'époque « orientalisante » du VIIIVIIe siècle av, J.-C. ${ }^{20}$. Les recherches entreprises à ce sujet afin de savoir laquelle de ces deux périodes correspond exactement au moment précis du contact font habituellement l'objet d'une controverse. Les thèmes traités par l'iconographie et les pièces d'une certaine époque découvertes dans d'autres communautés peuvent être des données extrêmement précieuses.

2.6. Il faut aussi se demander ce qu'une culture emprunte à une autre. Des phraséologies et certaines expressions peuvent être évidemment empruntées, mais il s'agit là d'une question très délicate où il est facile de tomber dans l'erreur. Il est ainsi frappant de constater la similitude de

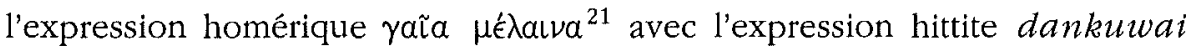
taknu, « la terre noire ${ }^{22}$. Mais en hittite dankuwai taknu ne désigne pas, comme chez Homère, la surface de la terre, mais le monde souterrain, le domaine qui correspond en grec à l'Hadès ou au Tartare ${ }^{23}$.

17 A. BERNABÉ, Un mito etiológico anatolio sobre el Taturo (CTH 16) en Nonno (Dion. 1.408s.), in Aula Orientalis, 6 (1988), p. 5-10.

18 Ces emprunts ont lieu aussi dans d'autres cultures : cf. pour les parallèles du mythe de Telipinou parmi les esvanes (un peuple du Caucasus), A. N. BENDUKIDZE, Xettskif mif o Telepinu $i$ ego suanskie Paralleli, in VDI (1973), p. 95-100, et pour les échos de Oullikoummi dans des récits phrygiens rapportés par Arnobe, W. BURKERT, Von Ullikummi zum Kaukasus: Die Felsgeburt des Unbolds. Zur Kontinuitat einer mündlicben Erzablung, in WilrzbJabrb., N.F., 5 (1979), p. 253-261.

19 C.H. GORDON, Homer and the bible, in Hebrew union Coll. Ann., 26 (1955), p. 43-108.

20 cf. M.L. WEST, op. cit. (n. 1), p. 28; W. BURKERT, Oriental and Greek... art. cit. (n. 2), p. 13.

21 HOM., Il., II, 699 al.

22 Cf. par exemple KUB XXXIII $120+119+$ XXXVI 31 Re I 14 (E. LAROCHE, Textes mytbologiques bittites en transcription, Paris, 1965, p. 40 [154]).

23 Mais cf. N. OETTINGER, Dle 'dunkle Erde' im Hetbitischen und Griechischen, in WO, 20-21 (1989-90), p. 83-98. 
Des noms peuvent aussi être empruntés; il est possible, par exemple, de

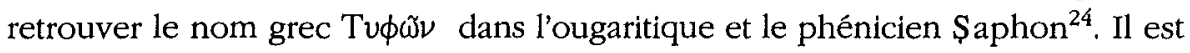
cependant plus courant de repérer des similitudes sur le plan des arguments, des thèmes ou des motifs.

2.7. Mondi $^{25}$ a récemment fait une proposition précise sur ce qui est transmis de l'Orient vers la Grèce. Il s'agirait des conceptual foci ${ }^{26}$, qu'il désigne sous le nom de mythic ideas. Les noms des dieux, précise-t-il, peuvent être représentés par des foyers conceptuels ou des noyaux auxquels s'ajoutent des fonctions et des thèmes narratifs. Les aspects qui à un. certain moment paraissent acceptables sont l'objet de diffusion.

2.8. À mon avis, le schéma de Mondi devrait être modifié. Les foyers conceptuels ne sont précisément pas les plus faciles à transmettre, vu qu'ils sont tirés du texte à travers une certaine vision des choses. Bien que la comparaison de ces foyers conceptuels ${ }^{27}$ s'avère être la démarche la plus intéressante dans l'analyse comparative, on ne peut pas dire pour autant qu'une telle mise en rapport fasse vraiment l'objet d'emprunts. Il en est évidemment de même (et là je suis de l'avis de Mondi) en ce qui concerne la façon de raconter une trame, le côté extérieur, l'aspect littéraire. Ce qui, à mon avis, voyage, ce sont certaines trames ou même ce que nous pouvons appeler des « segments de trame », des épisodes, des mythèmes, éléments qui expriment l'idée que quelqu'un fait quelque chose d'une certaine façon et dans un but précis. Plus il y aura de segments de trames empruntés ou plus l'articulation des éléments de la trame sera complexe, plus l'emprunt semblera évident. À ce sujet, il faut se passer de la distinction consistant à voir si ce qui est emprunté est représenté ou non par « le même mythe ».

Parfois les segments de trame sont simples: par exemple, dans l'Odyssée $e^{28}$, Eurykleia assied sur les genoux d'Autolykos son petit-fils qui vient de naître. Autolykos lui donne alors un nom, étymologiquement motivé :

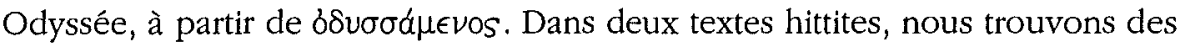
scènes très similaires, où le nouveau-né est posé sur les genoux du père et celui-ci lui donne de même un nom motivé; dans le Chant d'Oullikoummi, c'est Koumarbi qui l'appelle Oullikoummi, ce qui veut dire, " destructeur de

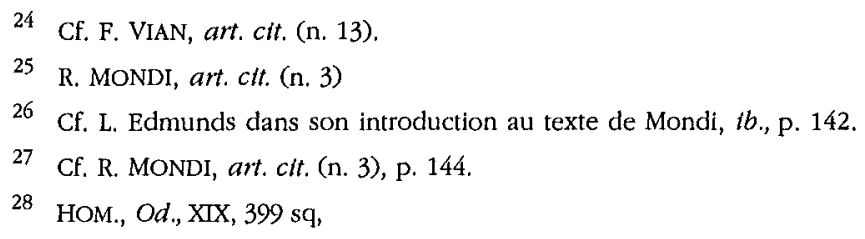


Koummiya », vu que c'est dans ce but qu'il l'avait engendré; dans le récit connu sous le nom de Récit d'Appou, Appou appelle son fils Méchant, vu les circonstances qui ont entouré sa naissance ${ }^{29}$. Ceci permet de mieux comprendre un passage d'Hésiode ${ }^{30}$ où Kronos se met à dévorer ses enfants :

Üs TLS ËKaOTOS

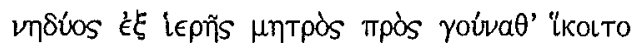

Pour West, c'est Rhéa qui dans le passage accouche à genoux ${ }^{31}$, mais si nous tenons compte du passage en question de L'Odyssée ainsi que d'autres ${ }^{32}$, il est évident qu'il ne s'agit pas des genoux de Rhéa, mais de ceux de Kronos, qui accueille les nouveau-nés sur ses genoux pour les légitimer et ensuite les dévorer.

Un exemple d'héritage de segments de trame complexes serait la Théogonie d'Hésiode, par rapport au Cycle de Koumarbi hittite.

Les segments de trame, qui dans une culture sont associés à un personnage précis, au moment de passer à une autre culture, sont associés à d'autres personnages qui présentent des fonctions analogues (par exemple, ceux concernant Koumarbi, à Kronos; ceux concernant 'Teshoub, à Zeus).

2.9. Un autre aspect très important reste à signaler : l'incorporation d'un segment de trame issu d'une culture étrangère à l'intérieur d'une œuvre littéraire produite dans un autre contexte culturel n'a pas lieu à l'état pur vu qu'elle subit nécessairement la contrainte des schémas culturels et littéraires de la culture qui adopte l'emprunt, ce qui, d'une part, fausse le sens général du thème et, d'autre part, rend moins reconnaissable sa propre présence ${ }^{33}$.

En d'autres termes : les segments de trame acquièrent dans chaque version du mythe un sens différent, qui est dû à plusieurs facteurs dont les plus importants sont les suivants : a) Syntaxe : la façon suivant laquelle se combinent les segments de trame entre eux et avec d'autres segments de trame; b) Fonction : l'usage donné au mythe : un mythe inséré dans un rituel est différent d'un mythe qui prétend simplement donner une certaine vision du monde; c) Intertextualité : le rapport du texte avec d'autres textes connus par l'auditeur. Rien que l'attribution des segments de trame à un personnage précis entraîne

Cf. H.A. HOFFNER, Bitth and name-giving in Hittite Texts, in JNES, 27 (1968), p. 198-203.

30

HÉs., $T h$., $459-460$.

31 WEST, op. cit. (n. 1), p. 294, ad v. 460.

32 Par exemple, HOM., Il., IX, 453 sq.

33 Cf. MOTTE, PIRENNE-DELFORGE, art. cit. (n. 4), p. 13 sq. 
toute une série d'associations fonctionnelles et significatives, qui varient totalement d'une culture à une autre; d) Situation : tout ce qui constitue le contexte culturel où sont plongés les auditeurs du mythe: les différents contextes culturels font en sorte que des séquences narratives similaires soient interprétées en des sens totalement différents ${ }^{34}$.

Ce qui est certain, c'est qu'à l'intérieur d'une culture, les segments narratifs tendent à se cristalliser sous forme d'éléments de plus en plus fixes, ils se standardisent, pour ainsi dire.

3. Aux difficultés théoriques que je viens sommairement d'exposer, et qui concernent l'établissement de points de départ méthodologiques admissibles, viennent s'ajouter un certain nombre de difficultés pratiques.

3.1. En premier lieu, les documents en langues orientales et les documents grecs sont traités dans des instituts de philologie traditionnellement séparés, du fait des difficultés inhérentes à la connaissance des différentes langues orientales, notamment le sumérien, l'akkadien, l'égyptien, le hittite, les langues indo-iraniennes, mais aussi le hourrite, l'éblaîte, et d'autres langues qui fournissent des textes intéressants. On ne peut aborder, pour être vraiment efficace, plus de deux ou trois domaines, ce qui oblige le philologue classique à n'avoir accès, comme source réelle de connaissance des littératures orientales (et vice versa), qu'à des traductions dont il ne peut vérifier la fidélité.

3.2. Cette séparation se manifeste également dans le fait que les chercheurs utilisent d'autres circuits de diffusion, d'autres publications, d'autres répertoires bibliographiques, et même d'autres bibliothèques. Un philologue classique se sent bien évidemment plus à l'aise dans les domaines abordés par L'Année Philologique que dans ceux figurant dans la bibliographie d'Orientalia. Une telle situation invite à encourager les travaux en équipe et à assurer une plus grande perméabilité dans l'accès à la bibliographie et aux publications. D'énormes progrès ont déjà été réalisés à ce sujet : colloques interdisciplinaires, publications d'études comparatives, éditions de textes avec traductions et commentaires accessibles aux non spécialistes.

4. Certaines difficultés sont relatives aux emprunts.

4.1. La première consiste à définir les critères permettant d'identifier un emprunt réel. Nous pouvons avoir recours à certains critères : l'un d'eux, appliqué depuis longtemps déjà, exige qu'il y ait un nombre élevé de coïnci- 
dences ou, plus simplement, une identité de détails très précis. Un autre critère tient compte de la présence d'inconséquences dans le texte. Lorsqu'un auteur essaie d'introduire dans son récit un élément étranger, il arrive que les sutures opérées ne passent pas inaperçues. J'ai pu étudier un cas assez intéressant dans un passage de l'Hymne bomérique à Déméter ${ }^{35}$.

4.2. Une deuxième difficulté est relative à la rigueur requise dans l'étude du texte oriental que nous prétendons comparer. Il est curieux de constater que des philologues classiques, capables de serrer de près le texte grec sur le plan philologique, ne soumettent pas aux mêmes exigences le texte oriental lui-même et le traitement philologique dont il a été l'objet. Ils tiennent a priori la traduction d'un texte oriental pour bonne, sans se demander si elle correspond au niveau des connaissances actuelles. Il est fréquent, par exemple, de trouver dans des travaux récents des références au livre de Pritchard ${ }^{36}$, dont les traductions datent pour la plupart des années cinquante et sont faites à partir d'éditions de textes évidemment antérieures. Une telle démarche est pourtant dangereuse, non seulement du fait que les progrès dans les domaines de la philologie akkadienne, hittite ou égyptienne, ont pu conduire à réinterpréter le sens de beaucoup de mots, et à altérer profondément l'interprétation de beaucoup de phrases du texte offert en $A N E T$, mais encore à cause des profonds changements qui ont pu se produire dans le texte lui-même, changements dus à la découverte de nouveaux fragments, ou au fait que les fragments anciens ont été reclassés, ou bien à la suite de nouvelles lectures. Ainsi, pour citer un autre exemple, si Vian dans son étude sur les origines orientales du mythe de Typhée ${ }^{37}$ avait pu disposer du texte du mythe d'Hedammou de Siegelová ${ }^{38}$, publié quelques années après, il aurait très certainement modifié quelques-unes de ses conclusions ${ }^{39}$.

En somme, le texte oriental qui fait l'objet de l'étude doit être connu dans sa version la plus moderne et la plus fiable; il convient en outre d'examiner le mieux possible le contexte mythique, religieux, idéologique et littéraire dans lequel le texte s'insère, ce qui suppose la consultation de la bibliographie

35 A. BERNABÉ, Himno a Deméter 43-46. Adaptactón de un motivo anatolio, in Emerita, 56 (1988), p. 87-93.

36 J.B. PRITCHARD (ed.), Ancient Near Eastern Texts relating to the Old Testament, Princeton, $1955^{2}, 1969^{3}$ (cit. souvent $A N E T$ ).

37 F. VIAN, art. cit. (n. 13).

38 J. SIEGELOVÁ, Appu Marchen und Hedammu Mythus, Wiesbaden, 1971.

39 Cf. A. BERNABÉ, La lucha contra el Dragón... 
secondaire, et, devant l'infranchissable obstacle représenté par la non connaissance de la langue, le recours à un expert s'impose souvent.

4.3. Une autre question difficile à prendre en compte dans l'étude comparative est de déterminer où et quand s'est produit le contact, dans la mesure où des éléments de réponse sont disponibles, par exemple, pour la localisation géographique de certaines versions du mythe qui ont pu se maintenir dans une zone locale, ou l'analyse de l'apparition du thème dans d'autres litteratures qui ont pu servir d'« intermédiaires » (comme la littérature phénicienne).

4.4. Il est important aussi de recourir à l'iconographie, élément auxiliaire toujours fondamental dans ces questions, mais relevant d'un univers dans lequel le philologue n'est d'ordinaire pas à l'aise. Aussi songera-t-il à faire appel, le cas échéant, à des spécialistes.

Le respect de ces exigences ne nous permettra pas, dans la plupart des cas, d'aller au-delà d'une hypothèse vraisemblable au sujet du mystérieux parcours qui a pu conduire certains récits de l'Orient, par delà les siècles et les espaces géographiques, dans une aire culturelle et linguistique aussi éloignée de la Grèce. Pareilles hypothèses sont impossibles à prouver strictement car les modalités de la transmission des textes de bouche à oreille nous échappent. En somme, c'est le plus souvent sur un terrain glissant que nous évoluons.

4.5. Il est encore, concernant l'étude des emprunts, une démarche importante: c'est l'analyse des différences entre les diverses versions présentant entre elles des analogies; il faut être en mesure d'apprécier la façon dont un même thème s'insère dans un ensemble mythique et culturel différent, à travers un réagencement de ses éléments par le biais des moyens les plus variés. C'est dans ce domaine que les recherches peuvent progresser, sur la base de données plus sûres, vu qu'elles s'appuient sur des éléments tangibles : les textes eux-mêmes.

Aux remarques déjà énoncées ${ }^{40}$ sur le sens différent que les segments de trame acquièrent dans chaque version du mythe, ajoutons encore quelques éléments utiles pour l'analyse.

a) Dans la mesure où ils sont des textes, les mythes possèdent une morphologie (éléments constitutifs), une syntaxe (agencement de ces éléments) et une sémantique (sens de chacun de ces éléments et de l'ensemble). Ceci appelle l'attention sur la façon dont les « segments de trame » s'agencent par de multiples procédés, soit entre eux, soit avec d'autres segments de trame 
apparaissant dans une version mais pas dans l'autre. On est amené à se demander si les mêmes éléments constitutifs, avec une syntaxe différente, acquièrent un sens distinct.

Il s'agit, en fait, d'établir la façon dont les éléments se structurent dans chaque version du mythe, structure d'opposition fondée sur différents éléments; de là vient qu'un mythe, tout en maintenant des analogies de forme et de fonction avec un autre, peut s'organiser autrement ou, au contraire, qu'une structure similaire peut servir à des fonctions très différentes.

C'est dire que la forme littéraire employée dans le récit du mythe à l'intérieur de chaque culture est également importante, vu qu'elle peut conditionner son sens. Un conte populaire (où on joue sur le côté anecdotique, le stratagème, la séparation nette entre ce qui est bon et ce qui est mauvais, le thème de la tromperie, les motifs classiques du folklore, etc.) est différent d'un mythe intégré dans un rituel (où peut prévaloir l'aspect étiologique et fonctionnel) ou d'un récit purement littéraire (ce qui implique un plus grand intérêt pour la forme ou la beauté du détail et, très souvent, l'adoption d'un style simplement allusif).

b) Il faut être aussi attentif à la fonction, à l'usage que l'on prête au mythe : quels aspects du mythe sont conditionnés, dans chaque version, par la fonction qui leur est propre ${ }^{41}$.

c) Un troisième élément de base est constitué par l'intertextualité : les rapports du texte avec d'autres textes connus par l'auditeur, c'est-à-dire avec d'autres variantes thématiques du même mythe ou avec d'autres noyaux mythiques qui peuvent avoir une influence sur le récit. Le poète a la possibilité de choisir certains segments narratifs et d'en écarter d'autres; il peut aussi opérer des combinaisons avec d'autres thèmes (ce que nous connaissons sous le nom de syncrétisme ${ }^{42}$.

d) Tenir compte de la situation s'avère aussi indispensable, à savoir ce qui constitue le contexte culturel dans lequel sont plongés les auditeurs du mythe : les différents contextes culturels font en sorte, en effet, que des séquences narratives similaires peuvent être interprétées dans des sens totalement différents.

41 À ce propos le livre classique est celui de G.S. KIRK, Mytb. Its meaning and function in Ancient and otber cultures, Berkeley-Los Angeles, 1970.

42 Sur la notion de syncrétisme, cf. MOTTE, PIRENNE-DELFORGE, art. cit. (n. 4), p. 15 sq. 
Il faut en particulier tenir compte de certains aspects fondamentaux, comme l'arrière-fond religieux, le modèle de société, voire certains éléments politiques.

1) En ce qui concerne l'arrière-fond religieux, il est fondamental de signaler les différences existantes entre les formes religieuses orientales (monothéisme hébreu, divinités essentiellement agraires, religions fortement étatisées ou non, conflits de divinités locales) et la religion grecque, polythéiste. Cet arrière-fond religieux peut conditionner une certaine transformation idéologique du mythe ${ }^{43}$.

2) Sont à prendre aussi en considération les influences culturelles : s'agit-il bien de sociétés agricoles, maritimes, commerçantes, de sociétés conflictuelles ou stables, de sociétés organisées sous un mode tribal, etc.

3) Interviennent encore les conditionnements politiques: contenus mythiques mis au service d'une cause politique, comme les différentes cosmogonies égyptiennes ou le développement et la configuration du mythe de Thésée à Athènes, au fil de l'instauration de la démocratie ${ }^{44}$.

4) En guise de conclusion, on observera que la recherche comparative, quand il s'agit de mythes génétiquement apparentés, présente un attrait supplémentaire : celui de nous offrir des exemples montrant comment un même matériau de base peut être modifié, ou mieux encore, la façon dont chaque communauté (ou même chaque auteur) a travaillé sur un mythe afin de l'adapter à ses propres besoins. On peut même aller plus loin et affirmer que c'est précisément l'analyse des différences entre les versions apparentées qui nous permet d'établir les raisons pour lesquelles le mythe a subi ces transformations.

L'analyse de ce qui a été enlevé ou ajouté à un mythe quelconque, comme celle des voies d'adaptation du mythe à un système de croyances ou d'intérêts est une aide précieuse pour éclairer le processus.

Une étude exhaustive de tous les mythes comparables fournirait sans doute des repères pour une interprétation générale des systèmes de croyances à l'œuvre dans les grandes cultures orientales et de la culture grecque.

43 J'ai exploré cette voie dans plusieurs travaux : A. BERNABÉ, Generactones de dioses $y$ sucesión interrumpida. El mito bitita de Kumarbi, la Teogonía de Hesiodo y el Papiro de Derveni, in Aula Orientalis, 7, 1989, p. 159-179; ID., La lucba contra el Dragón...; El viaje de los mitos: de Orlente a Occidente, in Actas de la Segunda semana canaria sobre el Mundo Antiguo: la Cultura del Viaje, La Laguna, sous presse; Los mitos de Hesíodo: origenes orientales, in A. BERNABÉ (ed.), Introducción a Hesíodo, Madrid, sous presse.

44 Cf. A. BERNABÉ, El mito de Teseo en la poesia arcaica y clásica, in R. OLmos (coord.), Coloquio sobre Teseo y la copa de Aison, Madrid, 1992, p. 97-118. 
Il faut ajouter que la recherche comparative permet de découvrir l'énorme richesse potentielle de ces thèmes et leur ouverture aux intérêts les plus variés, sans que leur identité se perde tout à fait. La survivance d'un mythe réside précisément dans son éternelle capacité d'adaptation.

Mais le plus important est que, dans la transmission des mythes, certains aspects négligés pendant des siècles dans les versions littéraires que nous connaissons peuvent suivre leur propre chemin (récits populaires, œuvres perdues, iconographie) et réapparaître à des époques fort tardives.

Une recherche méthodique, comme celle que je propose, permettra en outre d'atteindre un moyen terme entre la recherche effrénée de similitudes plus ou moins fortuites, fondée sur la conviction que « toute la littérature grecque était déjà en Orient », et la résistance obstinée à admettre la moindre influence étrangère sur les textes grecs. Un point de vue nuancé doit permettre de faire la part des choses et de distinguer ce qui est hérité de ce qui est propre à la littérature grecque.

Facultad de Filología

Alberto BERNABÉ

Universidad Complutense

E - 28040 MADRID 